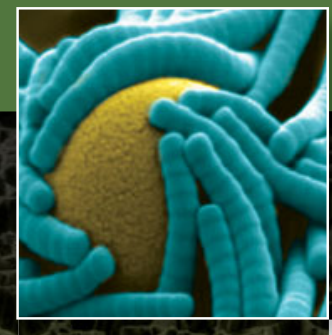

\title{
Patterning via self-organization and self-folding: Beyond conventional lithography
}

\author{
Sung Hoon Kang and Michael D. Dickey, Guest Editors
}

Conventional photolithography is an effective patterning technique that has enabled modern electronics and advanced micro- and nanoscale devices. However, it has limitations, including high cost, limited resolution, and poor compatibility with unconventional materials that may be soft, nonplanar, or difficult to process. There is active research ongoing to develop unconventional patterning methods such as self-organization and self-folding. Self-organization harnesses various driving forces to produce patterns without external intervention and includes methods such as self-assembly of block copolymers, capillary-driven assembly of micro-/ nanoscale structures, and thin-film instabilities. Self-folding (origami)-and its cousin, kirigami-harnesses patterning and materials strategies to convert planar substrates into three-dimensional shapes in response to external stimuli. These multidisciplinary approaches open many engineering opportunities by providing new and versatile material functionalities. This article overviews the field and the topics covered in the articles in this issue of MRS Bulletin, highlighting recent progress in patterning approaches based on self-organization and self-folding.

\section{Conventional lithography}

Photolithography is the most prevalent micro- and nanoscale patterning technique due to its effectiveness and efficiency. The process is illustrated in Figure 1. The term photolithography has a literal translation of "to write in stone using light," based on the Greek roots of the term. Typically, the "stone" is a silicon wafer coated with a light-sensitive polymer. Patterns of light (or electrons/ions) focused onto this coating change the solubility of the polymer in localized regions. The coating that remains after dissolving (i.e., developing) the soluble regions protects the substrate from subsequent processing, such as etching. The coating is therefore called a "photoresist," because it is designed to be sensitive to light and to resist etching. A pattern emerges on the substrate after etching the exposed regions and removing the photoresist.

Conventional photolithography is the cornerstone technology that enables fabrication in modern electronics. However, it also has many limitations, including (1) the need for a mask, focusing optics, light-sensitive materials, and expensive specialized equipment; (2) resolution limits defined by optics; and (3) poor compatibility with unconventional materials that may be soft, nonplanar, or difficult to process. Other "beam-based" lithography techniques, such as electron-beam lithography, can provide higher-resolution patterns than photolithography without the need for a mask, but otherwise, suffer similar limitations.

\section{Approaches to address the challenges of conventional lithography}

To address these issues, there have been numerous alternative (or "unconventional") approaches, ${ }^{1-3}$ including, for example, soft lithography, ${ }^{4}$ contact printing, ${ }^{5}$ dip-pen lithography, ${ }^{6}$ imprint lithography, ${ }^{7}$ and three-dimensional (3D) printing. ${ }^{8}$ No single method addresses all of the limitations of photolithography, and therefore, choosing a patterning method depends on the application, the materials, and the resources available.

Among these alternate methods, self-organization - the formation of patterns by harnessing forces across a range of scales - is an attractive option as it (1) forms complex patterns with minimal process monitoring and control, (2) can make structures beyond the resolution limit of photolithography, and (3) often provides more sustainable routes for fabrication by using ambient energy or minimizing environmental hazards. The contributions in this issue summarize key

Sung Hoon Kang, Department of Mechanical Engineering and Hopkins Extreme Materials Institute, Johns Hopkins University, USA; shkang@jhu.edu Michael D. Dickey, Department of Chemical and Biomolecular Engineering, North Carolina State University, USA; mddickey@ncsu.edu DOI: $10.1557 / \mathrm{mrs} .2016 .3$ 


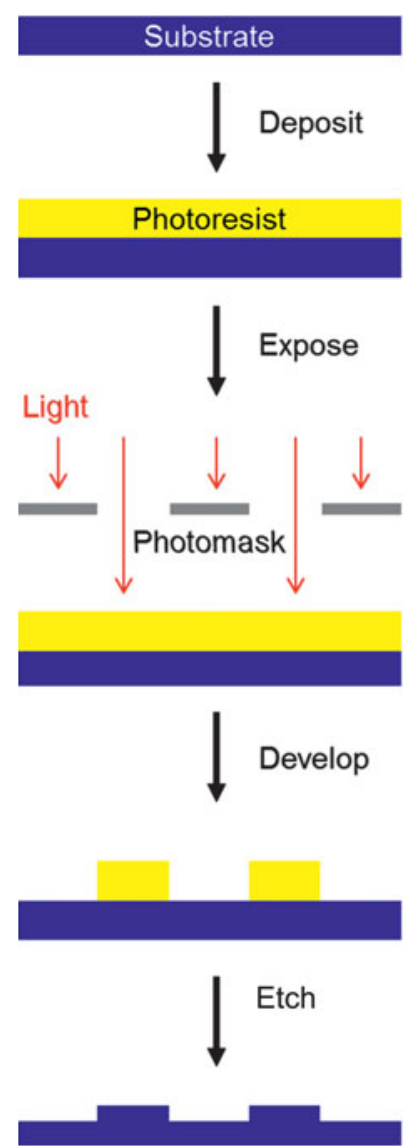

Figure 1. Conventional photolithography (and other "beambased" lithographies) focuses patterns of photons, electrons, or ions onto a substrate coated with a thin polymeric film called a photoresist. In the case of photolithography, the absorbed photons cause chemical reactions that change the solubility of the photoresist. Openings in the sacrificial photoresist locally expose the substrate to subsequent processing steps, such as etching. Credit: Ying Liu (North Carolina State University).

advances in self-organization using molecular-scale forces (block copolymer [BCP] self-assembly), capillary forces (capillary-induced assembly), and mechanical forces (instabilityinduced patterning).

In addition, this issue covers self-folding (origami) and kirigami as "pattern transforming" methods that give twodimensional (2D) patterns a greater degree of freedom to bend, extend, or fold. While conventional lithography is inherently planar, self-folding can create out-of-plane and 3D structures by harnessing responsive materials and managing mechanical stress. Kirigami utilizes strategic cuts in materials to allow otherwise rigid materials to extend significantly both in and out of plane.

Taken together, these new approaches open many scientific and engineering opportunities for micro- and nanoscale patterning by coupling disciplines, including physics, chemistry, biology, mechanics, and materials science, while providing new and versatile material functionalities. The field is truly multidisciplinary.

\section{Examples and applications}

To provide context, we offer several examples of selforganized and self-folded structures and their applications. For example, BCP self-assembly has been used for making structures beyond the resolution limit of optical lithography. BCPs are composed of two or more chemically distinct polymer segments. These segments self-assemble by phase-separating into small domains with geometries that can be tuned based on a number of factors, including the length and composition of the polymer. The resulting structures can be utilized as templates for high-density information storage, ${ }^{9}$ nanoelectronics, ${ }^{10}$ photonic crystals, ${ }^{11}$ and complex $3 \mathrm{D}$ structures (Figure $\left.2 \mathrm{a}-\mathrm{c}\right)^{12}$ that are difficult to make using conventional lithography.

Surface instabilities such as wrinkling, offer another approach to achieve patterning by self-organization. These instabilities form typically by minimizing energy between competing forces and have been used for applications such as controlled adhesion ${ }^{13}$ and wetting (Figure 2d-f), ${ }^{14}$ anti-biofouling, ${ }^{15}$ and tunable color. ${ }^{16}$

Capillary-induced self-organization can generate a range of structures based on the application of capillary forces to structures. Although capillary forces can self-assemble freestanding rigid structures by attracting floating objects (e.g., breakfast cereal in the "Cheerios effect"), ${ }^{17,18}$ here, we focus on capillary forces that deform micro-/nanostructures attached to a substrate. This approach, which allows greater structural complexity from features defined lithographically, has been utilized for microparticle trapping and release (Figure $2 \mathrm{~g}-\mathrm{h}),{ }^{19-22}$ molecule trapping and detection (Figure $2 \mathrm{i}-\mathrm{j}$ ), ${ }^{23}$ and whitening by surfaces that scatter light (Figure $2 \mathrm{~m}-\mathrm{n}){ }^{24}$

Self-folding has been used in various applications, including self-folding robots (Figure $3 \mathrm{a}-\mathrm{b}$ ), ${ }^{25}$ microgrippers (Figure $3 \mathrm{~d}-\mathrm{f}),{ }^{26}$ solar cells, ${ }^{27}$ and origami antennas. ${ }^{28}$ The use of strategic cuts in materials followed by folding (kirigami) enables, for example, the formation of stretchable electrodes for nonplanar devices (Figure 3c) ${ }^{29}$ and solar cells with integrated solar tracking. ${ }^{30}$

Structures formed by self-folding and self-organization can be classified based on their characteristic length scales, range of materials, and form factors, as summarized in Table I. For the form factors, mesostructure refers to a (surface-attached) structure approximately in the range of nanometer to micrometer scale. Film refers to planar features such as a coating or shell. Bulk refers to a mass of material typically bigger than the micrometer scale. These approaches cover a broad range of length scales and provide routes for making hierarchical structures. ${ }^{10}$ These methods are also compatible with diverse classes of materials and can be shaped into different forms.

\section{In this issue}

The articles in this issue of MRS Bulletin highlight and detail some of the recent progress in patterning approaches based on self-organization and self-folding. The article by Bai and Ross summarizes state-of-the-art applications of molecular selfassembly by controlling the composition and interactions of 

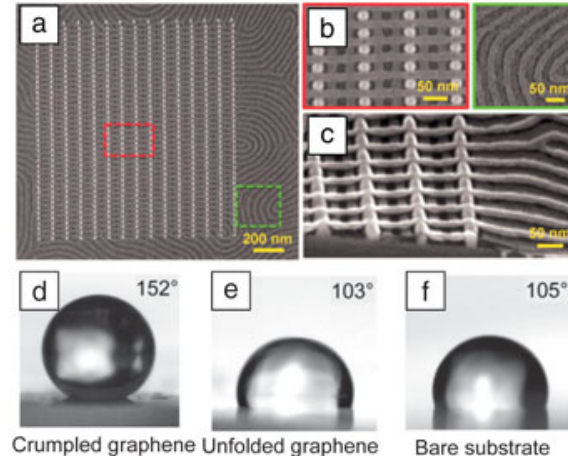

$103^{\circ}$

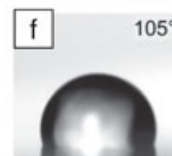

Crumpled graphene Unfolded graphene Bare substrate
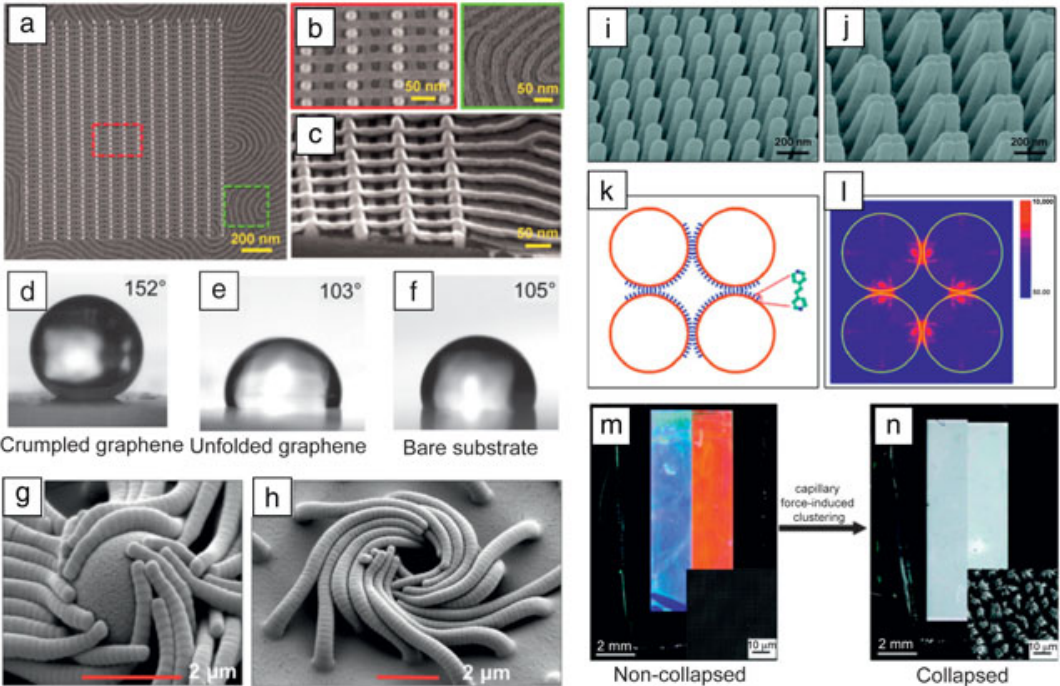

Non-collapse

Figure 2. Applications of different approaches. (a) Scanning electron microscope (SEM) image of an etched [( $\left.\left(\begin{array}{l}0 \\ 1\end{array}\right),(20)\right]$ bilayer structure on a substrate, (b) magnified SEM images (red and green borders refer to Figure $2 \mathrm{a}$ ), and (c) cross-sectional SEM image of the regions inside and outside the template. ${ }^{12}$ () 2012 American Association for the Advancement of Science. (d-f) Images showing the contact angle of a water drop: (d) $152^{\circ}$ on highly crumpled graphene, (e) $103^{\circ}$ on unfolded graphene, and (f) $105^{\circ}$ on bare polydimethylsiloxane substrate. ${ }^{14}$ (C) 2013 Nature Publishing Group. (g) A single polystyrene sphere trapped through the capillary-assisted conformal wrapping of epoxy nanobristles. (h) Coiled whirlpools remain after the removal of the spheres..$^{19}$ @) 2009 American Association for the Advancement of Science. (i-j) SEM images of fingers of gold-coated polymeric structures in (i) open position and (j) closed position after molecule trapping, driven by capillary interaction. (k) Schematic of molecules trapped in the nanogaps of the fingers, and (I) distribution of electric-field intensity (color bar scale) at $750 \mathrm{~nm}$ for four Au fingertips of $68-\mathrm{nm}$ radius. Reproduced with permission from Reference 23. (c) 2010 American Chemical Society. (m-n) Images of micropillar arrays (m) before and (n) after clustering. Two different colors result from Bragg diffraction of micropillar arrays with different periodicities, with clustering resulting in surface whitening. Reproduced with permission from Reference 24. (C) 2009 American Chemical Society.
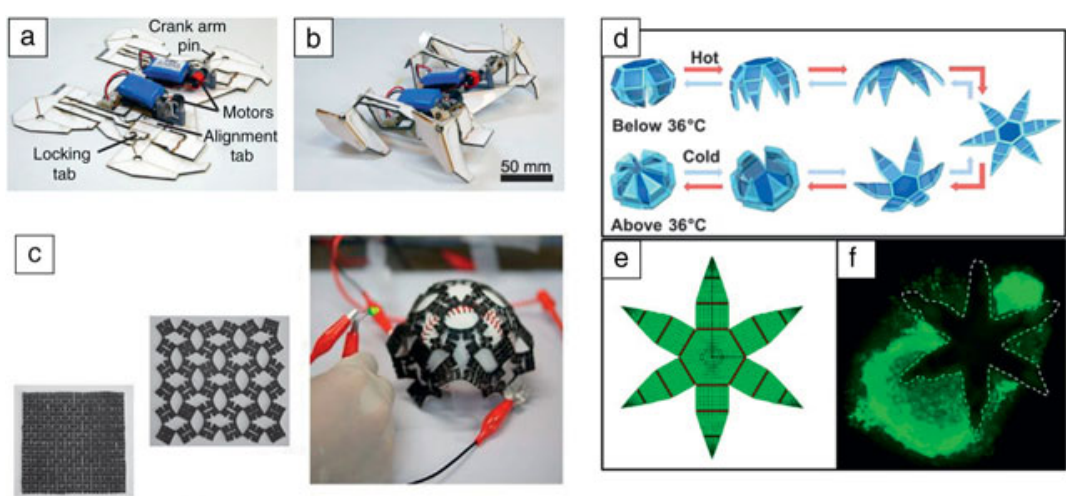

Figure 3. (a) A robot begins in a flat conformation, and folding is initiated 10 s after the batteries are connected. (b) Once the final folds cool and become rigid, the robot is assembled and ready for operation. Reproduced with permission from Reference 25. () 2014 American Association for the Advancement of Science. (c) A proof of concept for a stretchable electrode platform is shown by embedding fractal cuts in a silicone rubber sheet coated with a thin conducting film and conformably wrapping the structure around a spherical baseball without wrinkles. The green light-emitting diode remains lit after wrapping. Reproduced with permission from Reference 29. (c) 2014 National Academy of Sciences. (d) Schematic of the behavior of a self-folding microgripper, (e) schematic of a microgripper, and (f) capture and excision of cells from a live cell fibroblast clump (dyed green). Reproduced with permission from Reference 26. (c) 2015 American Chemical Society.
BCPs. This elegant approach to patterning is on the International Technology Roadmap for Semiconductors ${ }^{31}$ as a contender to replace or supplement conventional lithography for the fabrication of advanced electronics. Tawfick et al. illustrate ways to generate various 3D structures by self-assembly of filamentary materials through interactions among capillary, elastic, and adhesion forces. This approach enables formation of complex patterns that cannot be made using conventional lithography methods.

In their article, Wang and Zhao provide guidelines to control various topographic patterns by harnessing surface instabilities. This approach to patterning is noted for its simplicity and applicability to a wide range of length scales. Rogers et al. discuss self-folding to form out-of-plane, functional micro- and nanostructures. This approach allows transformation of 2D micro-/nanoscale structures into desired 3D shapes at length scales that are challenging for conventional fabrication tools. Finally, Yang et al. highlight the design and fabrication of super-conformable, foldable materials via fractal cuts and lattice kirigami. This approach enables otherwise rigid materials to extend significantly both in and out of plane.

\section{Summary}

Pattern formation by self-organization and selffolding provides unique opportunities for the materials community by addressing many of the issues associated with conventional lithography. With the exception of $\mathrm{BCPs}$, the methods described in this issue are not envisioned to compete with conventional lithography for fabricating state-of-the-art electronics. The methods discussed here generally lack the alignment and precision necessary for highyield processing of electronic materials. Instead, these new approaches typically seek to control and pattern diverse materials across a range of length scales at low cost in a way that gives rise to new functionalities. This brings up many interesting and challenging scientific questions, suggests new ways of designing and fabricating structures and devices, and enables applications previously unattainable. For example, kirigami enables stretchable structures from rigid materials, self-folding enables the formation of 3D structures from 2D structures, capillary assembly gives rise to dynamic structures, and instabilities create patterns at a low cost. As conventional lithography has empowered 
Table I. Length scales, materials, and forms covered by different approaches.

\begin{tabular}{|c|c|c|c|c|c|c|c|c|}
\hline \multirow[t]{2}{*}{ Approach } & \multicolumn{2}{|c|}{ Length Scales } & \multicolumn{3}{|c|}{ Materials } & \multicolumn{3}{|c|}{ Form } \\
\hline & $1 \mathrm{~nm}$ & $1 \mu \mathrm{m} 1 \mathrm{~mm}$ & Metal & Ceramic & Polymer & Mesostructure & Film & Bulk \\
\hline $\begin{array}{l}\text { Block copolymer } \\
\text { self-assembly }\end{array}$ & & & & & $\checkmark$ & $\checkmark$ & $\checkmark$ & \\
\hline $\begin{array}{l}\text { Elastocapillary } \\
\text { self-assembly }\end{array}$ & & & $\checkmark$ & $\checkmark$ & $\checkmark$ & $\checkmark$ & $\checkmark$ & $\checkmark$ \\
\hline Surface instability & & & $\checkmark$ & & $\checkmark$ & $\checkmark$ & $\checkmark$ & $\checkmark$ \\
\hline Self-folding & & & $\checkmark$ & $\checkmark$ & $\checkmark$ & & $\checkmark$ & \\
\hline Kirigami & & & $\checkmark$ & $\checkmark$ & $v$ & & $\checkmark$ & \\
\hline
\end{tabular}

9. J.Y. Cheng, C.A. Ross, V.Z.H. Chan, E.L. Thomas, R.G.H. Lammertink, G.J. Vancso, Adv. Mater. 13, 1174 (2001).

10. W.A. Lopes, H.M. Jaeger, Nature 414, 735 (2001).

11. A.C. Edrington, A.M. Urbas, P. DeRege, C.X. Chen, T.M. Swager, N. Hadjichristidis, M. Xenidou, L.J. Fetters, J.D. Joannopoulos, Y. Fink, E.L. Thomas, Adv. Mater. 13, 421 (2001).

12. K.G.A. Tavakkoli, K.W. Gotrik, A.F. Hannon, A. AlexanderKatz, C.A. Ross, K.K. Berggren, Science 336, 1294 (2012).

13. E.P. Chan, E.J. Smith, R.C. Hayward, A.J. Crosby, Adv. Mater. 20, 711 (2008).

14. J.F. Zang, S. Ryu, N. Pugno, Q.M. Wang, Q. Tu, M.J. Buehler, X.H. Zhao, Nat. Mater. 12, 321 (2013).

15. P. Shivapooja, Q.M. Wang, B. Orihuela, D. Rittschof, G.P. Lopez, X.H. Zhao, Adv. Mater. 25, 1430 (2013).

16. Q.M. Wang, G.R. Gossweiler, S.L. Craig, X.H. Zhao,

the materials community after decades of research and development, it is anticipated that self-organization and self-folding will provide many exciting opportunities beyond conventional lithography.

\section{Acknowledgments}

S.H.K. acknowledges start-up funds from the Whiting School of Engineering of the Johns Hopkins University. M.D.D. acknowledges support from the NSF (CAREER CMMI-0954321, ERC EEC-1160483, CMMI-1362284, DMR-1121107).

\section{References}

1. Y.N. Xia, J.A. Rogers, K.E. Paul, G.M. Whitesides, Chem. Rev. 99, 1823 (1999). 2. B.D. Gates, Q.B. Xu, J.C. Love, D.B. Wolfe, G.M. Whitesides, Annu. Rev. Mater. Res. 34, 339 (2004).

3. J.A. Rogers, H.H. Lee, Unconventional Nanopatterning Techniques and Applications (Wiley, Hoboken, NJ, 2009).

4. Y.N. Xia, G.M. Whitesides, Annu. Rev. Mater. Sci. 28, 153 (1998).

5. N.L. Jeon, K. Finnie, K. Branshaw, R.G. Nuzzo, Langmuir 13, 3382 (1997).

6. R.D. Piner, J. Zhu, F. Xu, S.H. Hong, C.A. Mirkin, Science 283, 661 (1999).

7. D.J. Resnick, S.V. Sreenivasan, C.G. Willson, Mater. Today 8, 34 (2005).

8. J.E. Smay, J. Cesarano, J.A. Lewis, Langmuir 18, 5429 (2002).

\section{Nat. Commun. 5, 4889 (2014).}

17. T.D. Clark, J. Tien, D.C. Duffy, K.E. Paul, G.M. Whitesides, J. Am. Chem. Soc. 123, 7677 (2001).

18. D. Vella, L. Mahadevan, Am. J. Phys. 73, 817 (2005)

19. B. Pokroy, S.H. Kang, L. Mahadevan, J. Aizenberg, Science 323, 237 (2009). 20. S.H. Kang, B. Pokroy, L. Mahadevan, J. Aizenberg, ACS Nano 4, 6323 (2010). 21. D. Wu, S.Z. Wu, S. Zhao, J. Yao, J.N. Wang, Q.D. Chen, H.B. Sun, Small $\mathbf{9}$, 760 (2013)

22. Y.L. Hu, Z.X. Lao, B.P. Cumming, D. Wu, J.W. Li, H.Y. Liang, J.R. Chu, W.H. Huang, M. Gu, Proc. Natl. Acad. Sci. U.S.A. 112, 6876 (2015).

23. M. Hu, F.S. Ou, W. Wu, I. Naumov, X.M. Li, A.M. Bratkovsky, R.S. Williams, Z.Y. Li, J. Am. Chem. Soc. 132, 12820 (2010).

24. D. Chandra, S. Yang, A.A. Soshinsky, R.J. Gambogi, ACS Appl. Mater. Interfaces 1, 1698 (2009).

25. S. Felton, M. Tolley, E. Demaine, D. Rus, R. Wood, Science 345, 644 (2014). D.H. Gracias, ACS Appl. Mater. Interfaces 7, 3398 (2015).

27. X.Y. Guo, H. Li, B.Y. Ahn, E.B. Duoss, K.J. Hsia, J.A. Lewis, R.G. Nuzzo, Proc. Natl. Acad. Sci. U.S.A. 106, 20149 (2009).

28. G.J. Hayes, Y. Liu, J. Genzer, G. Lazzi, M.D. Dickey, IEEE Trans. Antennas Propag. 62, 5416 (2014).

29. Y. Cho, J.H. Shin, A. Costa, T.A. Kim, V. Kunin, J. Li, S.Y. Lee, S. Yang, H.N. Han, I.S. Choi, D.J. Srolovitz, Proc. Natl. Acad. Sci. U.S.A. 111, 17390 (2014).

30. A. Lamoureux, K. Lee, M. Shlian, S.R. Forrest, M. Shtein, Nat. Commun. 6, 8092 (2015)

31. International Technology Roadmap for Semiconductors, http://www.itrs.net. $\square$ 26. J.C. Breger, C. Yoon, R. Xiao, H.R. Kwag, M.O. Wang, J.P. Fisher, T.D. Nguyen,

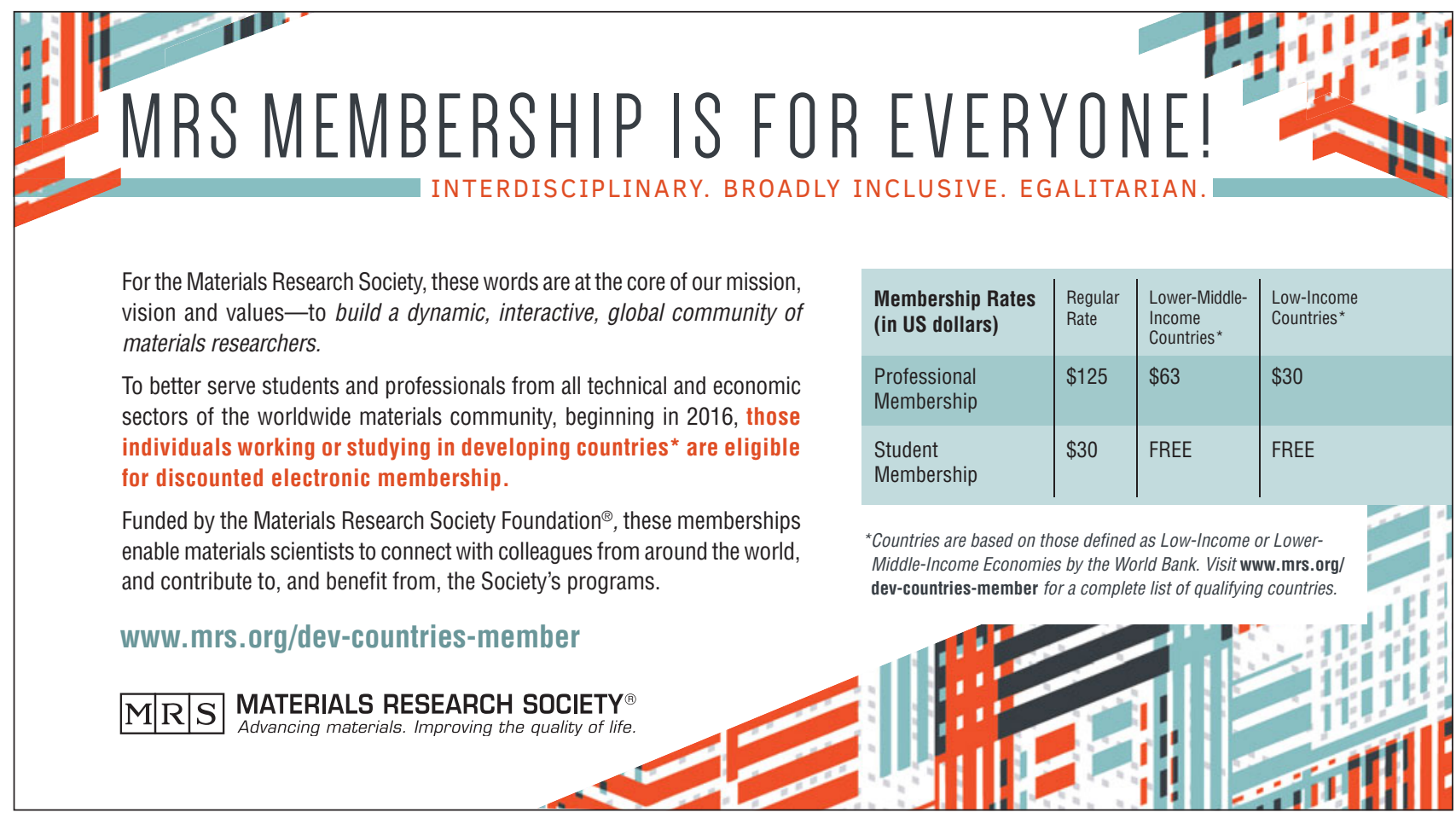

